\title{
Toward a systematic strategy for defining power counting in the construction of the energy density functional theory
}

\author{
C.-J. Yang, ${ }^{1}$ M. Grasso ${ }^{1}$ and D. Lacroix ${ }^{1}$ \\ ${ }^{1}$ Institut de Physique Nucléaire, CNRS/IN2P3, Université Paris-Sud, \\ Université Paris-Saclay, F-91406, Orsay, France
}

(Dated: September 27, 2018)

\begin{abstract}
We propose a new scheme for constructing an effective-field-theory-based interaction to be used in the energy-density-functional (EDF) theory with specific assumptions for defining a power counting. This procedure is developed through the evaluation of the equation of state (EOS) of symmetric and pure neutron matter going beyond the mean-field scheme and using a functional defined up to next-to-leading order (NLO), that we will call NLO EDF. A Skyrme-like interaction is constructed based on the condition of renormalizibility and on a power counting on $k_{F} / \Lambda_{h i}$, where $k_{F}$ is the Fermi momentum and $\Lambda_{h i}$ is the breakdown scale of our expansion. To absorb the divergences present in beyond mean-field diagrams, counter interactions are introduced for the NLO EDF and determined through renormalization conditions. In particular, three scenarios are explored and all of them lead to satisfactory results. These counter interactions contain also parameters which do not contribute to the EOS of matter and may eventually be determined through future adjustments to properties of some selected finite nuclei. Our work serves as a simple starting point for constructing a well-defined power counting within the EDF framework.
\end{abstract}




\section{INTRODUCTION}

The nuclear many-body problem has been extensively investigated since several decades. One of the challenges, at a very fundamental level, is the development of the nucleon-nucleon (NN) interaction. Several versions of phenomenological and recently developed chiral effective-fieldtheory (EFT) potentials have been applied to nuclear matter calculations through various ab-inito methods [1 22]. However, full convergence with respect to either the method or the version of the potential is not yet achieved. Moreover, although relevant progress was recently made to extend the area of applicability of ab-initio methods [23 30], it is not clear whether such methods can indeed be applied in future to the full nuclear chart, up to heavy nuclei. On the other hand, EDF theories have been adopted in nuclear many-body calculations for several decades with reasonable results [31]. In this approach, one does not start from the bare interaction between nucleons and assumes the validity of a mean-field (or beyond-mean-field) picture, in most cases constructed using effective phenomenological interactions. The Skyrme interaction [32, 33] is one of the most popular choices adopted in EDF. It consists of series of zero-range terms expanded in powers of momentum, which have an identical form (except for the density-dependent term) as the contact interactions present in pionless EFT [34, 35]. The success of Skyrme-based calculations in the EDF framework suggests that an EFT-like expansion based on a series of contact-type terms may exist, and results obtained at the mean-field level may be chosen to represent the leading-order (LO) contribution in such an expansion for EDF. ${ }^{1}$

To further explore along this direction, higher-order corrections need to be included. For example, in Refs. [37 40], the second-order contribution to the EOS of nuclear matter is derived analytically for Skyrme-type interactions. It is shown that, with the inclusion of a density-dependent term, a reasonable EOS can be obtained for matter up to second order at various isospin asymmetries after the divergence is subtracted in various ways. Furthermore, Ref. [41] shows that requirements based on renormalizability restrict the Skyrme interaction to have certain forms. In particular, only the $t_{0}$ or $t_{0}-t_{3}$ Skyrme-type interactions with some specific powers of the density $\alpha$ are allowed for the second-order EOS to be renormalizable. In practice, only the latter interaction $\left(t_{0}-t_{3}\right.$ model $)$ could provide an acceptable second-order EOS for symmetric matter. Note that, except for the finite part, contributions from second-order diagrams are regularization-scheme-

\footnotetext{
${ }^{1}$ Additional indication is provided in Ref. [36], where it is shown that the magnitude of various versions of Skyrme coefficients can be recovered by an expansion based on the unitarity limit.
} 
dependent. Whereas pionless EFT can be easily applied to vacuum or to dilute neutron matter and results become regularization-scheme independent after the renormalization is performed (for example, the free parameters can be matched to the effective-range expansion in the case of dilute neutron matter [42 48]), the renormalization/regularization process is more involved in the case of nuclear matter at larger densities. In particular, it is shown that if one considers symmetric nuclear matter at densities around the equilibrium point and starts with a Skyrme-like interaction, second-order results depend on the regularization procedure quite strongly [41]. In Ref. [41], the conventional definition of effective mass at the mean-field level was adopted, and no additional contact interactions were added (no counter terms).

In this work, we do not use a mean-field effective mass, we add counter terms by defining NLO effective interactions, and, as a consequence, we do not need to constrain the values of the density dependence $\alpha$. Starting from a $t_{0}-t_{3}$ model and the related contributions up to NLO, and guided by renormalizibility and renormalization-group (RG) analysis, we explore three types of possible counter terms and develop the EOS for symmetric and neutron matter up to NLO in EDF.

The structure of the present work is as follows. In section II, we describe the theoretical framework of our approach and report the LO results. In section III, we apply our method to develop a new Skyrme-like EFT interaction up to NLO and discuss the results. We summarize our findings in section IV.

\section{THEORETICAL FRAMEWORK}

\section{A. General considerations}

We first clarify the notation that we use in this work for LO and NLO.

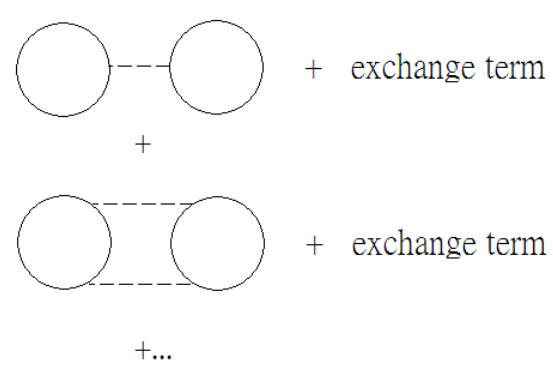

FIG. 1: Perturbative expansion of the ground-state energy in a uniform system. The diagramatic analysis of many-body perturbation theory is for instance illustrated in Ref. [49]. 


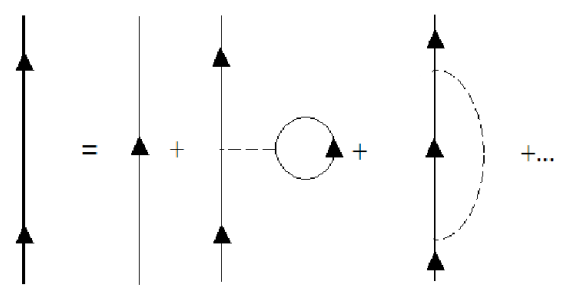

FIG. 2: Perturbative expansion of the exact Green's function. We refer to Ref. [49] for details on the diagrammatic representation of the many-body perturbation theory.

Starting from a given NN interaction, the EOS of nuclear matter can be evaluated by summing the diagrams of the perturbative expansion of the energy shown in Fig.1 The diagrams to obtain the dressed propagator $G$ (the exact Green's function) are shown in Fig. 2, Figures 1 and 2 represent the usual many-body perturbative expansion for the energy and the Green's function, respectively. In particular, the upper part of Fig. 1 describes the LO (first order or mean field) and the lower part the NLO (second order) of such a many-body expansion for the evaluation of the energy.

On the other hand, for very dilute neutron matter (densities $\rho<10^{-6} \mathrm{fm}^{-3}$ ), one can perform a perturbative calculation based on the effective-range expansion of the interaction, where higher loops are suppressed by higher powers of $a k_{F}, a$ being the neutron-neutron s-wave scattering length, and can obtain physical observables at very low densities [42 48]. However, most of nuclear systems of interest have a density $\rho$ much higher than the dilute limit. For example, typical densities in nuclear matter (of interest for finite nuclei) cover the range $\rho=0 \sim 0.3 \mathrm{fm}^{-3}$. To perform calculations at such densities one needs to use other procedures. A density-dependent neutron-neutron scattering length was for instance adopted in Ref. [50].

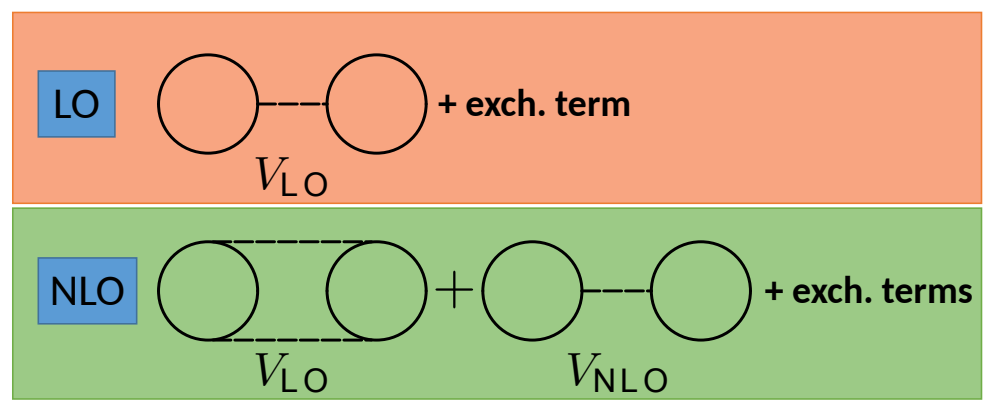

FIG. 3: The diagrammatic representation of contributions up to NLO for our EDF calculations.

If one assumes that particles move in an average mean field constructed from an effective 
interaction $V_{\text {eff }}$, only the upper diagram in Fig 1 (plus the exchange term) has to be evaluated for the computation of the energy. Generally, the parameters appearing in the effective interaction $V_{\text {eff }}$ are obtained by a fit to various nuclear properties such as binding energies and this adjustment is performed in most cases at the mean-field level. It is shown for example that a reasonable fit can be achieved for nuclear matter and some selected nuclei with a $V_{\text {eff }}$ of zero range (Skyrmelike interaction) or of finite range (Gogny interaction) [51, 52]. From an EFT point of view, this indicates that:

1. For densities of interest $\left(\rho=0-0.3 \mathrm{fm}^{-3}\right)$, there might exist an expansion to arrange diagrams in Figs. 1 and 2 order by order individually.

2. When inserting the propagator $G$, which contains the LO contribution from Fig. 2 into the LO diagram (Fig.1 upper part), the effect on the dressed propagator can be shifted to an effective interaction. One can thus define for instance an LO effective interaction, $V_{\mathrm{LO}}$, which is the one used to compute the LO contribution in Fig. 2 in the dressing of the operator.

To further improve the functional, NLO corrections must be considered. First, such corrections obviously include the second-order contribution (NLO in the sense of the many-body perturbative expansion) computed from $V_{\mathrm{LO}}$ by using the lower diagram in Fig 1, In addition, one may define an NLO effective interaction, $V_{\mathrm{NLO}}$ (that may be associated to the dressing of the propagator up to NLO in Fig. 2) and compute with such an interaction the energy contribution provided by the upper diagram in Fig,1. This defines an expansion related to our EDF calculations whose strategy is illustrated in Fig. 3, If only diagrams containing $V_{\mathrm{LO}}$ are retained in Fig. 3, such an expansion for EDF will of course coincide with the many-body expansion of Fig. 1. By proceeding in such a way, a next-to-next-to-leading order (NNLO) correction may then also be obtained, that contains at least the third-order contribution from $V_{\mathrm{LO}}$ and the mean-field energy contribution coming from $V_{\mathrm{NNLO}}$. The exact form of $V_{\mathrm{NLO}}$ and $V_{\mathrm{NNLO}}$ are to be decided by renormalizability conditions and power counting.

In this work, where the final EOS is to be evaluated using an NLO EDF, we label the interaction as $V_{\mathrm{LO}}$ if its second-order contribution in the perturbative many-body expansion is included in the final EOS, and as $V_{\mathrm{NLO}}$ if its mean-field contribution corresponds to NLO in the functional providing the EOS.

There are two features in our proposal. First, the parameters in the interaction are to be renormalized at each order. Second, the $V_{\text {eff }}$ constructed in this way is specifically designed for a 
beyond-mean-field framework where the independent-particle picture on which the mean field is based is completely lost. Corrections related to additional correlations such as for instance pairing correlations are not taken into account at the present stage.

To establish a power counting, some assumptions are necessary here. First, we arrange the interaction terms according to their contributions in powers of $k_{F}$ in the EOS. We denote the breakdown scale of our expansion as $\Lambda_{h i}$. Then, instead of on a dilute-limit expansion [43, 44], our power counting will be built on $\frac{k_{F}}{\Lambda_{h i}}$. We require that this expansion holds for $\rho=\rho_{L}-0.4 \mathrm{fm}^{-3}, \rho_{L}$ being the lowest density where a Skyrme-like interaction holds. To guarantee that $O\left(\left(\frac{k_{F}}{\Lambda_{h i}}\right)^{n+1}\right)$ contributes less than $O\left(\left(\frac{k_{F}}{\Lambda_{h i}}\right)^{n}\right)$, we fix a breakdown scale $\Lambda_{h i}$ so that $\Lambda_{h i}>k_{F}$. For the largest density that we consider for the validity of our expansion, $\rho=0.4 \mathrm{fm}^{-3}, \Lambda_{h i}$ should be larger than $2.3(1.8) \mathrm{fm}^{-1}$ for neutron (symmetric) matter. The fact that $O\left(\left(\frac{k_{F}}{\Lambda_{h i}}\right)^{n+1}\right)$ contributes less than $O\left(\left(\frac{k_{F}}{\Lambda_{h i}}\right)^{n}\right)$ should be confirmed by analyzing the power counting.

Second, since $V_{\mathrm{LO}}$ and $V_{\mathrm{NLO}}$ are not calculated directly in this work, it is preferable to make as least assumptions in the form of these interactions as possible. It is suggested in Ref. [41] that, to avoid a proliferation in the number of contact terms and at the same time have a reasonable fit of the EOS at LO, the preferable $V_{\mathrm{LO}}$ corresponds to a $t_{0}-t_{3}-$ like model. Then, throughout this work, our strategy is to utilize RG-analysis and renormalizability-check as tools to decide the structure of $V_{\mathrm{NLO}}$.

\section{B. Leading order for EDF}

The simplest form of interaction at LO in the momentum space contains $t_{0}\left(1+P_{\sigma} x_{0}\right)$ only, where $t_{0}$ and $x_{0}$ are free parameters and $P_{\sigma}=\left(1+\sigma_{1} \cdot \sigma_{2}\right) / 2$ is the spin-exchange operator. For pure neutron matter, a reasonable fit of EOS can be achieved by just one constant, that is the Bertsch parameter [53], which corresponds to the LO result from an expansion around the unitary limit $[36,54]^{2}$. However, this interaction fails to produce a reasonable fit for the EOS of symmetric matter at both mean-field level and with the second-order correction included $\left(\chi^{2}>1000\right.$ for both cases) [41]. Moreover, from the study of pionless EFT, it is established that the 3-body force is required at LO to avoid the triton from collapsing [55]. This suggests that, once symmetric matter is considered, a three-body force is required already at LO in the effective interaction. In the Skyrme

\footnotetext{
${ }^{2}$ Note that the Bertsch parameter is proportional to the kinetic term rather than the $t_{0}$ term in the Skyrme interaction.
} 
case, the collapse is avoided by introducing the so-called $t_{3}$ density-dependent two-body effective interaction. The next simplest form is a $t_{0}-t_{3}$-like model, which contains a density-dependent term, that is

$$
V_{\mathrm{LO}}=t_{0}\left(1+x_{0} P_{\sigma}\right)+\frac{t_{3}}{6}\left(1+x_{3} P_{\sigma}\right) \rho^{\alpha},
$$

and gives the mean-field EOSs for symmetric and neutron matter as

$$
\begin{gathered}
\frac{E_{S M}^{(\mathrm{LO})}}{A}=\frac{3}{10} \frac{k_{F}^{2}}{m}+\frac{1}{4} \frac{t_{0}}{\pi^{2}} k_{F}^{3}+\frac{1}{16} t_{3}\left(\frac{2}{3 \pi^{2}}\right)^{\alpha+1} k_{F}^{3 \alpha+3}, \\
\frac{E_{N M}^{(\mathrm{LO})}}{N}=\frac{3}{10} \frac{k_{F}^{2}}{m}+\frac{1}{12 \pi^{2}} t_{0}\left(1-x_{0}\right) k_{F}^{3} \\
+\frac{1}{24} t_{3}\left(1-x_{3}\right)\left(\frac{1}{3 \pi^{2}}\right)^{\alpha+1} k_{F}^{3 \alpha+3} .
\end{gathered}
$$

Note that we adopt here natural units $\hbar=c=1$. The subscripts SM and NM represent symmetric and neutron matter, respectively; $t_{0}, x_{0}, t_{3}, x_{3}$, and $\alpha$ are free parameters, $m$ is the nucleon mass, and $k_{F}=\left(3 \pi^{2} \rho / 2\right)^{1 / 3}\left(k_{F}=\left(3 \pi^{2} \rho\right)^{1 / 3}\right)$ for SM (NM). Note that, for $m$, one could choose to have it as an additional free parameter in principle, as done in Ref. [41]. Here, we adopt the point of view that all effects which modify the fermion propagator can be transferred order by order into $V_{\text {eff }}$ as an expansion in $\left(k_{F} / \Lambda_{h i}\right)^{n}$. Thus, the density-dependent part of the effective mass will be encoded into our effective potential, and $m=939 \mathrm{MeV}$ is adopted throughout this work.

We then perform best fits to determine the free parameters $\left(t_{0}, t_{3}, x_{0}, x_{3}, \alpha\right)$. The $\chi^{2}$ values are calculated as $\chi^{2}=\frac{1}{(N-1)} \sum_{i} \frac{\left(E_{i}-E_{i, r e f}\right)^{2}}{\Delta E_{i}^{2}}$, where $N$ is the number of points on which the adjustment is done, the sum runs over this number, $E_{i, r e f}$ is the benchmark value corresponding to the point $i$, and $\Delta E_{i}$ are all chosen equal to $1 \%$ of the reference value. In this work, we take $N=10$ (ten density values from 0 to $0.3 \mathrm{fm}^{-3}$ ), we choose as benchmark EOSs the mean-field SLy5 EOSs [56], and we perform a simultaneous fit to symmetric and pure neutron matter. The $\chi^{2}$ value is listed in Table@ together with the values of the parameters and the LO EOSs after fit are plotted in Fig. 4. As we can see, both EOSs (symmetric and neutron matter) are in quite reasonable agreement with the benchmark SLy5 mean-field curves [56]. In table [I, we compare the reference SLy5 values of the saturation density $\rho_{s}$, the incompressibility $K_{\infty}$ as well as the saturation energy $E\left(\rho_{s}\right) / A$ of symmetric matter to the values obtained at $\mathrm{LO}$ with the minimalist $t_{0}-t_{3}$ model. Except for the incompressibility, which is slightly overestimated, the reference EOS properties are rather well reproduced. 

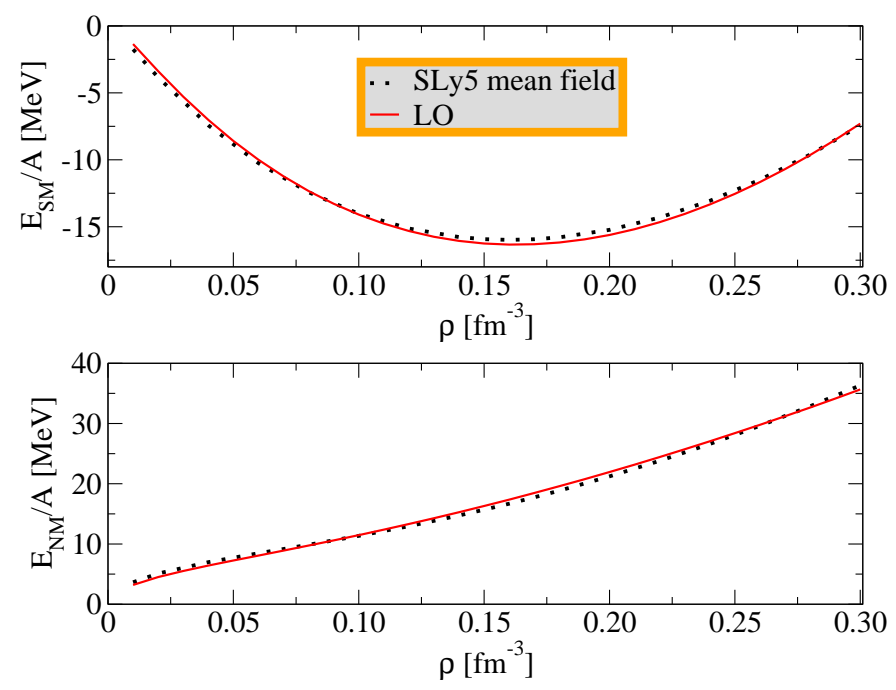

FIG. 4: Reference EOS as a function of the density $\rho$ obtained with the SLy5 functional (black dotted line) for symmetric (upper panel) and neutron (lower panel) matter. The LO EOSs (red solid line) are obtained by using Eqs. (2) and (3) with the parameters listed in Tables प.

\begin{tabular}{|c|c|c|c|c|c|}
\hline$\alpha$ & $t_{0}\left(\mathrm{MeV} \mathrm{fm}^{3}\right)$ & $t_{3}\left(\mathrm{MeV} \mathrm{fm}{ }^{3+3 \alpha}\right)$ & $x_{0}$ & $x_{3}$ & $\chi^{2}$ \\
\hline 0.4 & -1686 & 12096 & 0.2751 & 0.2530 & 77 \\
\hline
\end{tabular}

TABLE I: Parameter sets obtained by fitting the renormalized LO EOS on the SLy5 mean-field EOS.

\section{NEXT-TO-LEADING ORDER FOR EDF}

At NLO EDF one needs to consider the second-order corrections of the LO interaction and the first-order contribution from an NLO effective interaction. The latter will be determined based on renormalizability and $\mathrm{RG}$ analysis.

\begin{tabular}{|c|c|c|c|c|c|}
\hline & SLy5 & LO & $\mathrm{NLO}_{a b c}$ & $\mathrm{NLO}_{b c}$ & $\mathrm{NLO}_{c}$ \\
\hline$\frac{E\left(\rho_{s}\right)}{A}(\mathrm{MeV})$ & -16.18 & -16.31 & -15.93 & $-15.98 \pm 0.1$ & $-15.97 \pm 0.1$ \\
\hline$\rho_{s}\left(\mathrm{fm}^{-3}\right)$ & 0.162 & 0.162 & 0.16 & $0.16 \pm 0.003$ & $0.16 \pm 0.003$ \\
\hline$K_{\infty}(\mathrm{MeV})$ & 232.67 & 254.64 & 236.32 & $234.3 \pm 3.5$ & $233.2 \pm 3.7$ \\
\hline
\end{tabular}

TABLE II: Saturation density $\rho_{s}$, saturation energy $\frac{E\left(\rho_{s}\right)}{A}$ and incompressibility $K_{\infty}$ for symmetric matter provided by the SLy5 mean-field EOS, the $t_{0}-t_{3}$ model (LO) and our differents scenarios for NLO with three types of counter terms (see text). 


\section{A. NLO contribution of $V_{\mathrm{LO}}$ to the EDF}

The second-order corrections (many-body perturbative expansion) in the EOS for a $t_{0}-t_{3}$ LO effective interaction were evaluated in Refs. [37 39]. Here, we just report the results relevant for our LO interaction. The second-order corrections consist of three parts: (a) a finite part, $\frac{\Delta E_{f}^{(2)}\left(k_{F}\right)}{A}$; (b) a divergent part with a $k_{F}$-dependence already present at LO, $\frac{\Delta E_{a}^{(2)}\left(k_{F}, \Lambda\right)}{A}$; (c) a divergent part with a $k_{F}$-dependence not present at LO, $\frac{\Delta E_{d}^{(2)}\left(k_{F}, \Lambda\right)}{A}$. Here, $\Lambda$ is a sharp cutoff on the outgoing relative momentum $\vec{k}^{\prime}=\left(\vec{k}_{1}^{\prime}-\vec{k}_{2}^{\prime}\right) / 2$, with $\vec{k}_{1,2}^{\prime \prime}$ being the incoming (outgoing) momentum of nucleon 1,2. For symmetric matter, the second-order correction reads

$$
\frac{\Delta E_{S M, f}^{(2)}\left(k_{F}\right)}{A}=\frac{3 m}{\pi^{4}} \frac{[11-2 \ln 2]}{280} k_{F}^{4}\left[\begin{array}{c}
t_{0}^{2}\left(1+x_{0}^{2}\right)+2 t_{0} T_{3}\left(1+x_{0} x_{3}\right) k_{F}^{3 \alpha}+\left(1+x_{3}^{2}\right) T_{3}^{2} k_{F}^{6 \alpha}+ \\
\frac{3}{8} t_{0} T_{3} k_{F}^{3 \alpha} \alpha(3+\alpha)+\frac{3}{8} T_{3}^{2} k_{F}^{6 \alpha} \alpha(3+\alpha)+\frac{9}{256} T_{3}^{2} k_{F}^{6 \alpha} \alpha^{2}(3+\alpha)^{2}
\end{array}\right],
$$

$$
\begin{gathered}
\frac{\Delta E_{S M, a}^{(2)}\left(k_{F}, \Lambda\right)}{A}=-\frac{m}{8 \pi^{4}} \Lambda k_{F}^{3}\left[t_{0}^{2}\left(1+x_{0}^{2}\right)+2 t_{0} T_{3}\left(1+x_{0} x_{3}\right) k_{F}^{3 \alpha}+\frac{3}{8} t_{0} T_{3} \alpha(3+\alpha) k_{F}^{3 \alpha}\right], \\
\frac{\Delta E_{S M, d}^{(2)}\left(k_{F}, \Lambda\right)}{A}=-\frac{m}{8 \pi^{4}} \Lambda k_{F}^{3+6 \alpha} T_{3}^{2}\left[\left(1+x_{3}^{2}\right)+\frac{9}{256} \alpha^{2}(\alpha+3)^{2}+\frac{3}{8} \alpha(\alpha+3)\right],
\end{gathered}
$$

where

$$
T_{3}=\left(\frac{2}{3 \pi^{2}}\right)^{\alpha} \frac{t_{3}}{6}
$$

For neutron matter, one has

$$
\begin{gathered}
\frac{\Delta E_{N M, f}^{(2)}\left(k_{F}\right)}{A}=\frac{m}{\pi^{4}} \frac{[11-2 \ln 2]}{280} k_{F}^{4}\left[\left(T_{0}+k_{F}^{3 \alpha} T_{3}^{R}\right)^{2}\right], \\
\frac{\Delta E_{N M, a}^{(2)}\left(k_{F}, \Lambda\right)}{A}=-\frac{m}{24 \pi^{4}} \Lambda k_{F}^{3}\left[T_{0}^{2}+2 T_{0} T_{3}^{R} k_{F}^{3 \alpha}\right], \\
\frac{\Delta E_{N M, d}^{(2)}\left(k_{F}, \Lambda\right)}{A}=-\frac{m}{24 \pi^{4}} \Lambda k_{F}^{3+6 \alpha}\left[\left(T_{3}^{R}\right)^{2}\right]
\end{gathered}
$$

where

$$
\begin{aligned}
T_{0} & =t_{0}\left(1-x_{0}\right) \\
T_{3}^{R} & =\left(\frac{1}{3 \pi^{2}}\right)^{\alpha}\left[\frac{t_{3}}{6}\left(1-x_{3}\right)+\frac{1}{48} t_{3}\left(1-x_{3}\right) \alpha(3+\alpha)\right] .
\end{aligned}
$$


The contribution from the rearrangement terms [35, 57] is included in the above equations. A summary of the different $k_{F}$ dependences in the EOS is shown in table III.

\begin{tabular}{cllll}
\hline \hline Contribution to $E / A$ & $V_{\mathrm{LO}}$ & $V_{\mathrm{NLO}}^{(a)}$ & $V_{\mathrm{NLO}}^{(b)}$ & $V_{\mathrm{NLO}}^{(c)}$ \\
\hline Mean field & $k_{F}^{3}, k_{F}^{3+3 \alpha}$ & $k_{F}^{3}$ & $k_{F}^{3+3 \alpha}$ & $k_{F}^{3+6 \alpha}$ \\
\hline Second-order & $k_{F}^{4}, k_{F}^{4+3 \alpha}, k_{F}^{4+6 \alpha}$ & & & \\
& $k_{F}^{3}, k_{F}^{3+3 \alpha}$ & & & \\
& $k_{F}^{3+6 \alpha}$ & & & \\
& & & \\
\hline \hline
\end{tabular}

TABLE III: Different $k_{F}$ dependences in the EOSs of neutron and symmetric matter obtained for different interactions discussed in the text. All the terms in black are cutoff independent. In the second-order contribution of $V_{\mathrm{LO}}$, the terms in red and blue are linearly cutoff dependent. In particular, the terms in blue can be treated either by absorbing them in the mean-field part by a redefinition of the parameters or by introducing counter terms of the type $V_{\mathrm{NLO}}^{(a)}$ and $V_{\mathrm{NLO}}^{(b)}$. The terms in red in the second-order contribution correspond, in the absence of restrictions on the $\alpha$ values, to terms that require explicitly the introduction of counter terms, $V_{\mathrm{NLO}}^{(c)}$. Note finally that the second-order contributions of $V_{\mathrm{NLO}}$ are not shown since they will appear only when going to higher orders.
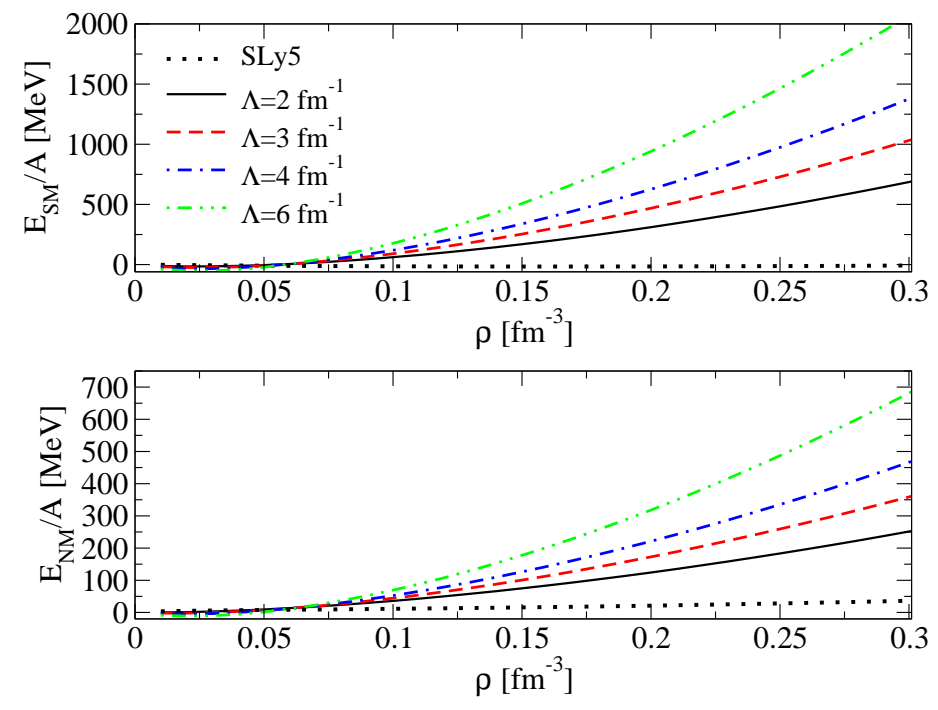

FIG. 5: EOS as a function of the density $\rho$ for symmetric (upper panel) and neutron (lower panel) matter. The dotted line represents the mean-field SLy5 EOSs. The LO parameters listed in Table \are used to compute the EOSs generated from the NLO EDF using $V_{\mathrm{LO}}$. 


\section{B. Scenarios for regularization}

In Fig. 5 we plot the unrenormalized EOSs obtained by including the contributions generated from the NLO EDF using the $V_{\mathrm{LO}}$ interaction, where we simply use the LO parameters listed in Table \. As one can see, both EOSs show strong cutoff dependence and, as the increase of $\Lambda$, depart further away from the benchmark value. This shows that renormalization is required.

The separation of the second-order part into three contributions is at the heart of the strategy we use below to propose different scenarios for regularisation. Let us first start with preliminary remarks that are important for the coming discussion:

- Except for the finite parts (Eqs. (4) and (86)), the exact forms of $\frac{\Delta E_{a}^{(2)}}{A}$ and $\frac{\Delta E_{d}^{(2)}}{A}$ (Eqs. (15)-(6) and Eqs. (9)-(10) ) are regularization-scheme dependent. However, for our expansion to make sense, the final EOS should not depend on a particular scheme after proper renormalization. This will be verified in the following by comparing the effect of various counter terms.

- The parameter $\alpha$, which appears in the density-dependent term, requires a special attention because each value of $\alpha$ would provide a different $k_{F}$ dependence. In the present work, we keep $\alpha$ as a free parameter in the renormalization.

- The highest $k_{F}$-dependence appearing in the second-order EOS is $k_{F}^{4+6 \alpha}$. Thus, by a simple counting in powers of $k_{F}$, the $t_{1}$ and $t_{2}$ terms of the Skyrme interaction (which contribute at first order as $k_{F}^{5}$ in the EOS) do not enter in the NLO effective interaction for $\alpha<\frac{1}{6}$. In the following, since $\alpha$ is varied freely, $\alpha$ might exceed $\frac{1}{6}$. In this case, one should keep in mind that, a priori, one has also to include the $t_{1}$ and $t_{2}$ terms of the Skyrme interaction.

In a previous study [41], it was shown that the divergence appearing in $\frac{\Delta E_{a}^{(2)}}{A}$ may be absorbed by a redefinition of the existing parameters since those terms have the same $k_{F}$ dependence as in first-order terms. For the divergence appearing in $\frac{\Delta E_{d}^{(2)}}{A}$, one could first search for some special values of $\alpha$ which would give for $\frac{\Delta E_{d}^{(2)}}{A}$ the same $k_{F}$-dependences as those appearing in the meanfield part. Then, one could perform the renormalization by absorbing the $\Lambda$-divergence into a redefinition of the parameters. This approach was adopted in Ref. [41], where no new counter terms were included.

In this work, we adopt a more general approach. We release the requirement on specific values of $\alpha$ and, in general, we allow treating $\frac{\Delta E_{a}^{(2)}}{A}$ and $\frac{\Delta E_{d}^{(2)}}{A}$ in the same way: both divergences present in $\frac{\Delta E_{a}^{(2)}}{A}$ and $\frac{\Delta E_{d}^{(2)}}{A}$ may be directly renormalized by NLO EDF contributions. This allows us to use 
the divergence generated at NLO by an LO interaction as an important guide for the construction of an NLO effective interaction, denoted by $V_{\mathrm{NLO}}$. In principle, each $\Lambda k_{F}^{n}$-divergence in the EOS can be directly associated with an NLO counter part $A_{n} k_{F}^{n}$, where $A_{n}$ denotes an additional free parameter ${ }^{3}$. A term in the effective interaction of the form of $O\left(\left(\vec{k}-\vec{k}^{\prime}\right)^{n-3 v-3} \rho^{v}\right)$ will contribute as $O\left(k_{F}^{n}\right)$ in the EOS, where $v$ is an arbitrary number which satisfies $n-3 v-3=$ even number. Note that the parameter $v$ does not appear in the EOS of matter. This additional free parameter may eventually be adjusted with a fit to reproduce properties of finite nuclei. Interactions of the above type appear naturally for example when one expands the terms coming from a resumed expression [60 63]].

Without fixing $\alpha$ to specific values, the minimum counter term required to absorb the divergences present in $\frac{\Delta E_{d}^{(2)}}{A}$ is the one proportional to $k_{F}^{3+6 \alpha}$. On the other hand, the divergence present in $\frac{\Delta E_{a}^{(2)}}{A}$ can be absorbed by just a redefinition of $t_{0}, x_{0}, t_{3}$, and $x_{3}$ or by adding more counter terms proportional to $k_{F}^{3}$ and $k_{F}^{3+3 \alpha}$. Note that, in both cases, the mean-field values of the parameters are modified.

The three contact interactions which correspond to the three divergences appearing $\frac{\Delta E_{a}^{(2)}}{A}$ and $\frac{\Delta E_{d}^{(2)}}{A}$ can be written as

$$
\begin{aligned}
& V_{\mathrm{NLO}}^{(a)}=a\left(1+P_{\sigma} x_{a}\right) f_{a}\left(\left(\vec{k}-\vec{k}^{\prime}\right)^{-3 v_{a}}, \rho^{v_{a}}\right), \\
& V_{\mathrm{NLO}}^{(b)}=b\left(1+P_{\sigma} x_{b}\right) f_{b}\left(\left(\vec{k}-\vec{k}^{\prime}\right)^{3 \alpha-3 v_{b}}, \rho^{v_{b}}\right), \\
& V_{\mathrm{NLO}}^{(c)}=c\left(1+P_{\sigma} x_{c}\right) f_{b}\left(\left(\vec{k}-\vec{k}^{\prime}\right)^{6 \alpha-3 v_{c}}, \rho^{v_{c}}\right),
\end{aligned}
$$

where $f_{a, b, c}$ are functions which contain infrared regulators to prevent potential singularities at $\rho \rightarrow 0$ or $\left|\vec{k}-\overrightarrow{k^{\prime}}\right| \rightarrow 0$; it may turn out that a best fit to finite nuclei would provide negative powers for $\left(\vec{k}-\vec{k}^{\prime}\right)$ or $\rho$. Away from singularities, we have $f_{a, b, c}\left(\left(\vec{k}-\vec{k}^{\prime}\right)^{n-3 v-3}, \rho^{v}\right) \approx\left(\vec{k}-\vec{k}^{\prime}\right)^{n-3 v-3} \rho^{v}$. $a, b, c, x_{a}, x_{b}, x_{c}$ are free parameters to be determined by an adjustment of the EOS. On the other hand, $v_{a}, v_{b}$ and $v_{c}$ are extra parameters that could be determined only through further adjustments done for finite nuclei. With their mean-field contribution directly entering in the NLO EOS, the above three counter terms provide $k_{F}^{3}, k_{F}^{3 \alpha+3}$, and $k_{F}^{6 \alpha+3}$ terms to the EOS (see table III). Note that only Eq. (15) (with contribution $k_{F}^{6 \alpha+3}$ ) is necessarily required by renormalizability. The effect of the other two terms (Eqs. (13) and (14)) can be replaced by readjusting the values of $t_{i^{\prime}} s$ and $x_{i^{\prime} s}$ so that these two counter terms, for nuclear matter, should just modify the values of the parameters and not the power counting.

\footnotetext{
${ }^{3}$ A recent approach which constructs the interaction directly on a particular power series of $\sum_{n} k_{F}^{n}$ is introduced in Refs. [58, 59]. However, in present work we consider $n$ to be any real number.
} 
In table IV, we list all Skyrme-type $V_{\mathrm{LO}}$ and $V_{\mathrm{NLO}}$ interactions and the $k_{F}$ dependencies generated in the EOS from the LO and NLO EDFs. We show in red the $V_{\mathrm{NLO}}$ contributions which are not included in the present study because we limit $\alpha$ to be less than $1 / 6$.

\begin{tabular}{|c|c|c|}
\hline \hline Skyrme-type interaction & $k_{F}$-dep. in the EOS from LO EDF & $k_{F}$-dep. in the EOS from NLO EDF \\
\hline$V_{\mathrm{LO}}: t_{0}\left(1+x_{0} P_{\sigma}\right)$ & $k_{F}^{3}$ & $t_{0}^{2}$ terms: $k_{F}^{3}, k_{F}^{4}$ \\
\hline$V_{\mathrm{LO}}: t_{3}\left(1+x_{3} P_{\sigma}\right) \rho^{\alpha}$ & $k_{F}^{3+3 \alpha}$ & $t_{3}^{2}$ terms: $k_{F}^{3+6 \alpha}, k_{F}^{4+6 \alpha}$ \\
\hline & & $t_{0} t_{3}$ terms: $k_{F}^{3+3 \alpha}, k_{F}^{4+3 \alpha}$ \\
\hline$V_{\mathrm{NLO}}($ counter terms): Eq. (13) & & $k_{F}^{3}$ \\
\hline$V_{\mathrm{NLO}}($ counter terms): Eq. (14) & & $k_{F}^{3+3 \alpha}$ \\
\hline$V_{\mathrm{NLO}}($ counter terms): Eq. (15) & $k_{F}^{3+6 \alpha}$ \\
\hline$V_{\mathrm{NLO}}: t_{1}\left(1+x_{1} P_{\sigma}\right)\left(\mathbf{k}^{\prime 2}+\mathbf{k}^{2}\right)$ & & $k_{F}^{5}$ \\
\hline$V_{\mathrm{NLO}}: t_{2}\left(1+x_{2} P_{\sigma}\right) \mathbf{k}^{\prime} \cdot \mathbf{k}$ & & $k_{F}^{5}$ \\
\hline
\end{tabular}

TABLE IV: Skyrme-type $V_{\mathrm{LO}}$ and $V_{\mathrm{NLO}}$ interactions and $k_{F}$ dependencies generated in the EOS from the LO and NLO EDFs. We show in red the $V_{\mathrm{NLO}}$ contributions which are not included in the present study because we limit $\alpha$ to be less than $1 / 6$. Note that, here, we do not include spin-orbit and tensor interactions that should a priori appear as $V_{\mathrm{NLO}}$ and contribute at EDF NLO with their mean-field functional. The reason is that such mean-field contributions are zero in infinite matter.

The scenario we consider for regularization will depend on the type of counter terms that are included in $V_{\mathrm{NLO}}$. Since the case with no counter term has already been discussed in Ref. [41], we consider three possible scenarios, referred as scenario (c), (bc) and (abc) that refers to the fact that only $V_{\mathrm{NLO}}^{(c)}$, only $V_{\mathrm{NLO}}^{(b)}$ plus $V_{\mathrm{NLO}}^{(c)}$, or all three counter terms are used to construct $V_{\mathrm{NLO}}$, respectively. The resulting EOSs will be respectively called EOS-NLO, EOS-NLO $b c$ and EOS-NLO $a b c$.

Scenario (abc): Adopting all three types of counter terms, the EOS up to NLO reads

$$
\frac{E_{S M}^{(\mathrm{NLO})}\left(k_{F}\right)}{A}=\frac{3}{10} \frac{k_{F}^{2}}{m}+\frac{k_{F}^{3}}{4 \pi^{2}}\left[t_{0}+A\right]+\frac{k_{F}^{3 \alpha+3}}{4 \pi^{2}}\left[T_{3}+B\right]-\frac{m}{8 \pi^{4}} k_{F}^{3+6 \alpha} C+\frac{\Delta E_{S M, f}^{(2)}\left(k_{F}\right)}{A}
$$

for symmetric matter and

$$
\begin{aligned}
\frac{E_{N M}^{(\mathrm{NLO})}\left(k_{F}\right)}{A}= & \frac{3}{10} \frac{k_{F}^{2}}{m}+\frac{1}{12 \pi^{2}}\left[t_{0}\left(1-x_{0}\right)+A^{*}\right] k_{F}^{3}+\left[\frac{1}{24} t_{3}\left(1-x_{3}\right)\left(\frac{1}{3 \pi^{2}}\right)^{\alpha+1}+\frac{B^{*}}{4 \pi^{2}}\right] k_{F}^{3 \alpha+3} \\
& -\frac{m}{8 \pi^{4}} k_{F}^{3+6 \alpha} C^{*}+\frac{\Delta E_{N M, f}^{(2)}\left(k_{F}\right)}{A}
\end{aligned}
$$

for neutron matter. Note that, to simplify the notation, we have defined $A^{(*)}, B^{(*)}$, and $C^{(*)}$ as the parameters originating from Eqs. (13), (14), and (15) for symmetric (neutron) matter. The 


\begin{tabular}{cccccccc}
\hline \hline$\alpha$ & $t_{0}\left(\mathrm{MeV} \mathrm{fm}^{3}\right)$ & $t_{3}\left(\mathrm{MeV} \mathrm{fm}^{3+3 \alpha}\right)$ & $x_{0}$ & $x_{3}$ & $A\left(\mathrm{MeV} \mathrm{fm}^{3}\right) B\left(\mathrm{MeV} \mathrm{fm}^{3+3 \alpha}\right) C\left(\mathrm{MeV} \mathrm{fm}^{3+6 \alpha}\right)$ \\
\hline-0.083 & 307.6 & 97.27 & -2.721 & -13.31 & -7329 & 8339 & 14965 \\
\hline \hline$A^{*}\left(\mathrm{MeV} \mathrm{fm}^{3}\right)$ & $B^{*}\left(\mathrm{MeV} \mathrm{fm}^{3+3 \alpha}\right)$ & $C^{*}\left(\mathrm{MeV} \mathrm{fm}^{3+6 \alpha}\right)$ & $\chi^{2}$ & & & \\
\hline-24149 & 11159 & 18781 & 0.46 & & & \\
\hline \hline
\end{tabular}

TABLE V: Parameter sets obtained by fitting the renormalized second-order EOS to the SLy5 mean-field EOS. Here the second-order EOSs reported in Eqs. (16) and (17) are used.

parameters $a, b$ and $c$ in Eqs. (13), (14), and (15) can be splitted into two parts. One cancels the linear $(\Lambda)$ divergence in the EOS. The remaining parts are finite, are denoted by $A^{(*)}, B^{(*)}$, and $C^{(*)}$ and enter into the fitting procedure.

No cutoff is present in Eqs. (16) and (17) because all possible divergences have been absorbed by counter terms. We then perform the renormalization by refitting Eqs. (16) and (17) to a benchmark symmetric and neutron matter EOS, given by the SLy5 Skyrme interaction at the mean-field level, from $\rho=0 \sim 0.3 \mathrm{fm}^{-3}$. In Fig. 6, we plot the resulting EOS for symmetric and neutron matter up to $\rho=0.4 \mathrm{fm}^{-3}$. As one can see, both the fit in symmetric and neutron matter agree with the standard value with $\chi^{2}=0.46$ as listed in Table V. However, it is not possible to perform a RG-analysis in this case because no cutoff-dependence is present in the final EOS.
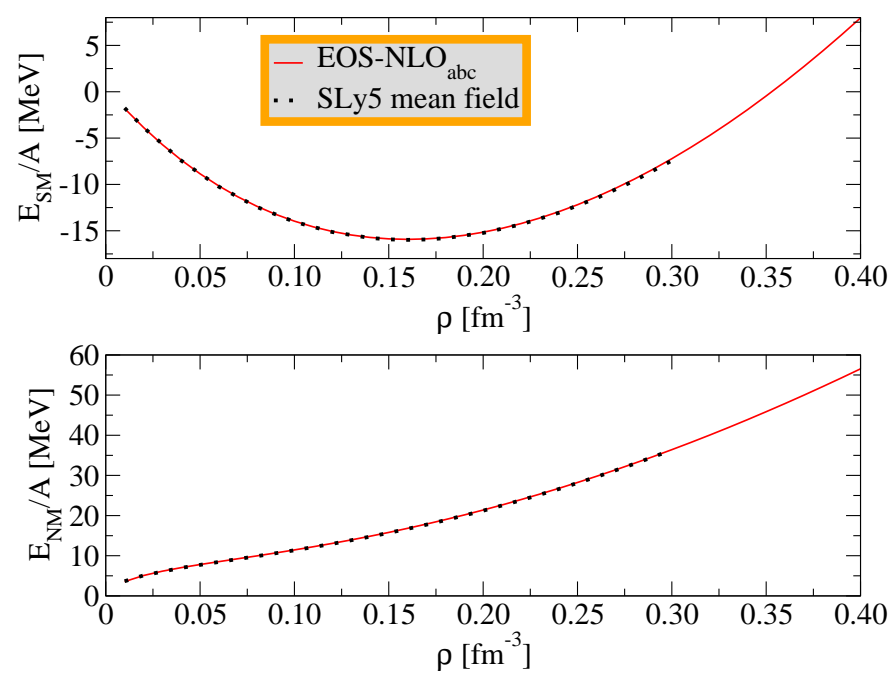

FIG. 6: EOS as a function of the density $\rho$, where the subscript SN (NM) represents symmetric (neutron) matter. The dotted (red solid) line represents the mean-field SLy5 (renormalized NLO) EOSs. The NLO EOSs are obtained by using the scenario (abc), that is Eqs. (16) and (17), with the parameters listed in Table $\mathrm{V}$

Scenario (bc): Next, we renormalize the second-order EOS in the absence of the $a$-counter 
term (Eq. (13) ) and let the $k_{F}^{3}$-divergence be absorbed by a redefinition of the parameters. The resulting EOS reads

$$
\frac{E_{S M}^{(\mathrm{NLO})}\left(k_{F}, \Lambda\right)}{A}=\frac{3}{10} \frac{k_{F}^{2}}{m}+\frac{k_{F}^{3}}{4 \pi^{2}} t_{0}^{\Lambda}+\frac{k_{F}^{3 \alpha+3}}{4 \pi^{2}}\left[T_{3}+B\right]-\frac{m}{8 \pi^{4}} k_{F}^{3+6 \alpha} C+\frac{\Delta E_{S M, f}^{(2)}\left(k_{F}\right)}{A}
$$

for symmetric matter and

$$
\begin{aligned}
\frac{E_{N M}^{(\mathrm{NLO})}\left(k_{F}, \Lambda\right)}{A}= & \frac{3}{10} \frac{k_{F}^{2}}{m}+\frac{k_{F}^{3}}{12 \pi^{2}} t_{0}^{\Lambda}\left(1-x_{0}^{\Lambda}\right)+\left[\frac{1}{24} t_{3}\left(1-x_{3}\right)\left(\frac{1}{3 \pi^{2}}\right)^{\alpha+1}+\frac{B^{*}}{4 \pi^{2}}\right] k_{F}^{3 \alpha+3} \\
& -\frac{m}{8 \pi^{4}} k_{F}^{3+6 \alpha} C^{*}+\frac{\Delta E_{N M, f}^{(2)}\left(k_{F}\right)}{A}
\end{aligned}
$$

for neutron matter. Here, $t_{0}^{\Lambda}$ and $x_{0}^{\Lambda}$ are

$$
\begin{aligned}
t_{0}^{\Lambda} & =t_{0}-\frac{m \Lambda}{2 \pi^{2}} t_{0}^{2}\left(1+x_{0}^{2}\right), \\
t_{0}^{\Lambda}\left(1-x_{0}^{\Lambda}\right) & =t_{0}\left(1-x_{0}\right)-\frac{m \Lambda}{2 \pi^{2}} t_{0}^{2}\left(1-x_{0}\right)^{2} .
\end{aligned}
$$

Note that, through $t_{0}^{\Lambda}$ and $x_{0}^{\Lambda}, \Lambda$ is present in Eqs. (18) and (19). However, together with Eqs. (20) and (21), it is clear that the cutoff dependence in the final EOS can always be eliminated properly $^{4}$ after the renormalization is done. We then perform again a best fit to the mean-field SLy5 EOS (from $\rho=0 \sim 0.3 \mathrm{fm}^{-3}$ ), for $\Lambda=1.2-20 \mathrm{fm}^{-1}$. The resulting EOSs for symmetric and neutron matter are plotted in Fig.7, and the parameters and corresponding $\chi^{2}$ are listed in Table VI.

\begin{tabular}{|c|c|c|c|c|c|c|c|c|c|c|}
\hline$\Lambda\left(\mathrm{fm}^{-1}\right)$ & 2 & 4 & 6 & 8 & 10 & 12 & 14 & 16 & 18 & 20 \\
\hline$t_{0}\left(\mathrm{fm}^{2}\right)$ & -2.804 & 2.024 & -1.146 & 4.443 & 1.415 & 1.206 & -1.960 & 2.627 & -0.6473 & 0.4415 \\
\hline$t_{3}\left(\mathrm{fm}^{2+3 \alpha}\right)$ & 31.89 & -28.99 & -4.159 & -47.48 & 2.661 & 18.31 & -15.53 & -0.5724 & -20.38 & -21.44 \\
\hline$x_{0}$ & -2.229 & 1.350 & 1.095 & 0.6359 & 1.448 & 1.202 & 1.203 & -0.1834 & 4.257 & 0.3196 \\
\hline$x_{3}$ & -1.463 & $2.059 \cdot 10^{-3}$ & -6.376 & 0.1812 & -11.70 & -0.7088 & -0.9565 & 31.64 & -1.103 & -0.4495 \\
\hline$B\left(\mathrm{fm}^{2+3 \alpha}\right)$ & 14.54 & -29.11 & -23.61 & 65.71 & -4.749 & -16.44 & 50.99 & 29.71 & 39.75 & -28.14 \\
\hline$C\left(\mathrm{fm}^{2+6 \alpha}\right)$ & -2.713 & -146.3 & -93.89 & 67.63 & -46.00 & -59.68 & 97.23 & 51.99 & 37.24 & -104.2 \\
\hline$B^{*}\left(\mathrm{fm}^{2+3 \alpha}\right)$ & 28.67 & 37.49 & 10.77 & 17.46 & 7.152 & 19.17 & 4.072 & 25.15 & 32.86 & 8.702 \\
\hline$C^{*}\left(\mathrm{fm}^{2+6 \alpha}\right)$ & 73.58 & 160.3 & 49.08 & 69.76 & 44.66 & 96.88 & 20.12 & 73.83 & 114.1 & 39.76 \\
\hline$\alpha$ & $4.77 \cdot 10^{-2}$ & $1.48 \cdot 10^{-2}$ & $3.13 \cdot 10^{-2}$ & $2.28 \cdot 10^{-2}$ & $3.59 \cdot 10^{-2}$ & $1.68 \cdot 10^{-2}$ & $4.96 \cdot 10^{-2}$ & $6.48 \cdot 10^{-2}$ & $1.92 \cdot 10^{-2}$ & $3.44 \cdot 10^{-2}$ \\
\hline$\chi^{2}$ & 0.39 & 2.19 & 0.76 & 0.88 & 2.41 & 4.04 & 1.95 & 3.62 & 1.67 & 1.18 \\
\hline
\end{tabular}

TABLE VI: Parameter sets obtained by fitting the renormalized second-order EOS to the SLy5 mean-field EOS. Here the second-order EOSs reported in Eqs. (18) and (19) are used.

\footnotetext{
${ }^{4}$ After renormalization, one is left with a residual cutoff-dependence of the order $\Re\left(\Lambda, k_{F}, \Lambda_{h i}\right)\left(\frac{k_{F}}{\Lambda_{h i}}\right)^{n+1}$, where $\Re$ is a function of the natural size and $n$ is the order of the calculation [64].
} 


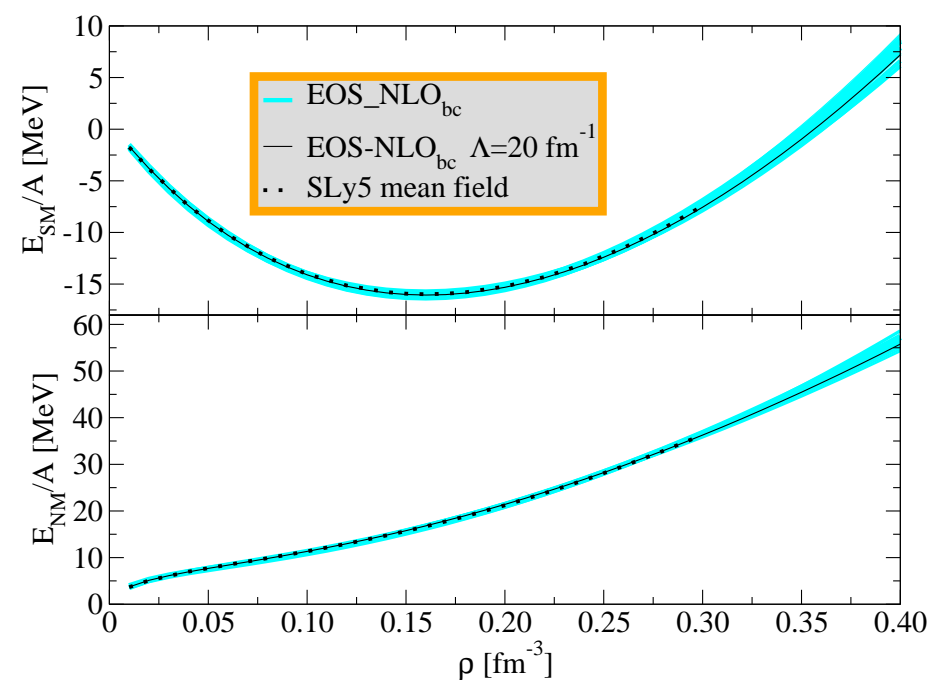

FIG. 7: (EOS as a function of the density $\rho$, where the subscript SN (NM) represents symmetric (neutron) matter. The dotted line (blue band) represents the mean-field SLy5 (renormalized NLO) EOSs. The NLO EOSs are obtained by using the scenario (bc), that is Eqs. (18) and (19) with the parameters listed in Table VI] Here, the cutoff is taken in the window $\Lambda=1.2-20 \mathrm{fm}^{-1}$ and the errorbars correspond to the cutoff dependence of the fit.

Scenario (c): For the case where one only allows the minimum counter term to enter, that is the counter term in Eq. (15), the divergences in powers of $k_{F}^{3}$ and $k_{F}^{3 \alpha+3}$ are absorbed into a redefinition of $t_{0}, x_{0}, t_{3}$, and $x_{3}$, and the resulting EOS reads

$$
\begin{aligned}
\frac{E_{S M}^{(\mathrm{NLO})}\left(k_{F}, \Lambda\right)}{A}= & \frac{3}{10} \frac{k_{F}^{2}}{m}+\frac{k_{F}^{3}}{4 \pi^{2}} t_{0}^{\Lambda}+\frac{k_{F}^{3 \alpha+3}}{4 \pi^{2}} T_{3}-\frac{m}{8 \pi^{4}} k_{F}^{3+6 \alpha} C \\
& -\frac{m}{8 \pi^{4}} \Lambda k_{F}^{3+3 \alpha} t_{0}\left[2 T_{3}\left(1+x_{0} x_{3}\right)+\frac{3}{8} T_{3} \alpha(3+\alpha)\right]+\frac{\Delta E_{S M, f}^{(2)}\left(k_{F}\right)}{A}
\end{aligned}
$$

for symmetric matter and

$$
\begin{aligned}
\frac{E_{N M}^{(\mathrm{NLO})}\left(k_{F}, \Lambda\right)}{A}= & \frac{3}{10} \frac{k_{F}^{2}}{m}+\frac{k_{F}^{3}}{12 \pi^{2}} t_{0}^{\Lambda}\left(1-x_{0}^{\Lambda}\right)+\frac{k_{F}^{3 \alpha+3}}{24} t_{3}\left(1-x_{3}\right)\left(\frac{1}{3 \pi^{2}}\right)^{\alpha+1} \\
& -\frac{m \Lambda k_{F}^{3+3 \alpha}}{12 \pi^{4}} T_{0} T_{3}^{R}-\frac{m}{8 \pi^{4}} k_{F}^{3+6 \alpha} C^{*}+\frac{\Delta E_{N M, f}^{(2)}\left(k_{F}\right)}{A}
\end{aligned}
$$

for neutron matter. Here, only the $C^{(*)}$ counter term enters into play. Again, renormalizability is guaranteed as the divergences can always be absorbed into a redefinition of the Skyrme parameters. With the same renormalization strategy as for the previous two cases, the resulting EOSs for symmetric and neutron matter are plotted in Fig. 8, and the parameters and corresponding $\chi^{2}$ are listed in Table VII. Note that for $\Lambda>4 \mathrm{fm}^{-1}$, some of the values of $\alpha$ exceed $1 / 6$. In principle, one should thus include the mean-field contributions from the $t_{1}, t_{2}$-terms in the EOS in these 


\begin{tabular}{|c|c|c|c|c|c|c|c|c|c|c|}
\hline$\Lambda\left(\mathrm{fm}^{-1}\right)$ & 2 & 4 & 6 & 8 & 10 & 12 & 14 & 16 & 18 & 20 \\
\hline$t_{0}\left(\mathrm{fm}^{2}\right)$ & -2.987 & -2.543 & -2.140 & -2.543 & -1.725 & 1.885 & -0.7193 & -1.362 & 1.512 & 1.407 \\
\hline$t_{3}\left(\mathrm{fm}^{2+3 \alpha}\right)$ & 19.36 & -0.9911 & -4.586 & 20.02 & 0.8675 & 1.645 & 10.39 & -0.4202 & 0.7416 & 2.174 \\
\hline$x_{0}$ & 1.291 & 0.6370 & 0.8911 & 0.7149 & 0.5508 & 0.5239 & 2.247 & 0.6074 & 0.4943 & 0.5695 \\
\hline$x_{3}$ & -0.1825 & -15.17 & -4.145 & -0.6774 & 12.87 & -7.037 & $-6.189 \cdot 10^{-3}$ & -25.80 & -12.45 & -4.917 \\
\hline$C\left(\mathrm{fm}^{2+6 \alpha}\right)$ & 12.01 & -3.791 & 1.962 & 20.95 & -1.383 & -3.364 & 0.3387 & -0.8847 & -3.547 & -3.286 \\
\hline$C^{*}\left(\mathrm{fm}^{2+6 \alpha}\right)$ & 10.64 & 61.689 & 0.7346 & 33.21 & -2.972 & -3.140 & -3.027 & -3.430 & -3.773 & -3.953 \\
\hline$\alpha$ & $6.88 \cdot 10^{-2}$ & 0.170 & 0.126 & $4.20 \cdot 10^{-2}$ & 0.224 & 0.187 & 0.226 & 0.223 & 0.205 & 0.190 \\
\hline$\chi^{2}$ & 3.01 & 1.06 & 1.25 & 2.53 & 0.34 & 0.55 & 1.63 & 0.55 & 1.92 & 1.23 \\
\hline
\end{tabular}

TABLE VII: Parameter sets obtained by fitting the renormalized second-order EOS to the SLy5 mean-field EOS. Here the second-order EOSs reported in Eqs. (22) and (23) are used.

cases. We have performed such calculations and found that including non-zero $t_{1}, t_{2}$-terms does not improve the overall quality of the fits.

So far, we have checked three out of the four possible scenarios for the NLO contact terms. We do not consider the possibility of having an $(a c)$ scenario because the NLO EOS is unlikely to consist of counter terms proportional to $k_{F}^{3}$ and $k_{F}^{3+6 \alpha}$, without the intermediate term $k_{F}^{3+3 \alpha}$.

From the fact that satisfactory fits (with similar quality) can be obtained by all three scenarios, we conclude that the regularization-scheme dependence present in Eqs. (5)-(6) and Eqs. (9)-(10) does not affect the NLO results after renormalization. The differences due to the regularization scheme can be transferred into the counter terms present in Eqs. (13) and (14). The independence of the final result of the regularization scenario is also illustrated in Table $\amalg$ where we see that the properties of symmetric matter are almost independent of the scenario and well match the reference SLy5 EOS.

Although in the present work the interactions are treated perturbatively and the small difference between the LO and NLO EOSs suggests that the power counting should be straightforward, the fact that the LO interaction $V_{\mathrm{LO}}$ is not derived from an underlying microscopic theory and the presence of $V_{\mathrm{NLO}}$ counter terms leave the whole theory into the danger that what is generated could be nothing but just another phenomenologically fitted functional. Therefore, an EFT-based power counting analysis is necessary. For an explicit determination of the power counting, a RG-analysis needs to be performed first. Here, we performed a RG-analysis for the two scenarios where the cutoff dependence is still present. The cutoff dependence at the density $\rho=0.4 \mathrm{fm}^{-3}$ is plotted as a function of the cutoff $\Lambda$ in Figs. 9 and 10, where the EOSs are obtained by Eqs. (18)-(19) and Eqs. (22)-(23), respectively. In addition, the running of parameters is plotted as a function of 
cutoff $\Lambda$ in Fig. 11 and Fig. 12 for scenario (bc) and (c), respectively. Note that the adjustment is performed up to $\rho=0.3 \mathrm{fm}^{-3}$, so the results at $\rho=0.4 \mathrm{fm}^{-3}$ are predictions. As one can see, the cutoff dependence is reduced at higher $\Lambda$ in both cases. In addition, a similar convergence pattern is observed. However, due to the uncertainty generated by the large number of parameters (nine for the case in Fig. 9 and seven for the case in Fig. 10), the convergence patterns in both cases are not quite smooth. This might give rise to potential problems in performing a full power counting analysis as introduced, for example, in Ref. [64]. Nevertheless, such an analysis is still of interest and should be performed at NLO and NNLO level to give further confirmation to our approach. We leave it as a future work. Finally, for $\alpha \geq \frac{1}{6}$, one needs to consider also the meanfield contributions coming from the $t_{1}$ and $t_{2}$ terms. They contribute at LO as $\frac{\theta_{s}}{4 \pi^{2}} k_{F}^{5}$ and $\frac{\theta_{s}-\theta_{v}}{4 \pi^{2}} k_{F}^{5}$ to the EOS of symmetric and pure neutron matter, respectively, where

$$
\begin{array}{r}
\theta_{s}=\frac{1}{10}\left[3 t_{1}+t_{2}\left(5+4 x_{2}\right)\right], \\
\theta_{v}=\frac{1}{10}\left[t_{1}\left(2+x_{1}\right)+t_{2}\left(2+x_{2}\right)\right] .
\end{array}
$$

We have repeated the fit for $\alpha \geq \frac{1}{6}$ for the above three cases. However, we found that, despite the presence of four additional parameters, the $\chi^{2}$ increases for most of the cutoff values in the three cases for $V_{\mathrm{NLO}}$. This suggests that the inclusion of the $t_{1}, t_{2}$ terms should be deferred to NNLO.

A final point we wish to stress is that the sets of parameters listed in Tables VIVII] are obtained through a fit to the SLy5 EOS of symmetric and pure neutron matter with 10 points ranging from $\rho=0-0.3 \mathrm{fm}^{-3}$. We have changed the number of points from 9 to 12 and found that the parameters are stable with respect to the number of fitting points. However, due to the large number of parameters and to the fact that the fits are performed only to two EOSs, there exist other sets of parameters which generate slightly $(<1 \%)$ larger $\chi^{2}$. Thus, we cannot guarantee that the parameters listed in Tables VFVII are the final values to be used in all applications. A full determination of parameters is only possible with a general fit to both nuclear matter and finite nuclei, which we defer to a future work. Nevertheless, when another set of parameters (with slightly larger $\chi^{2}$ ) is adopted, we observed that the convergence pattern as listed in Figs. 9 and 10 is unchanged, that is, the oscillation with respect to the cutoff $\Lambda$ becomes smaller at higher $\Lambda$. Also, after canceling the divergence by the contact terms, it could be possible to keep a subset of parameters cutoff invariant. For example, one could try to keep $t_{3}, x_{3}$, and $\alpha$ cutoff invariant in the scenario (bc) and $\alpha$ cutoff invariant in the scenario (c). Decreasing the number of parameters for the fit might indeed help to reduce the fluctuations seen in Figs. 9-12. This kind of test will be performed in a future work to gain more insight toward establishing an EFT-based functional. 


\section{CONCLUSIONS}

We have proposed a new approach to generate an effective interaction up to NLO in the EDF framework. Two tools from EFT, renormalizability and RG-analysis, are utilized to construct and analyze the new effective interaction. Under the condition that the renormalizability is guaranteed, we explored three possible scenarios for the NLO counter terms. We found that all three scenarios

produce second-order EOSs with similar quality, which indicates that our EOS up to NLO is independent of the regularization scheme. Benchmark symmetric and neutron matter EOSs can be reproduced in our approach within $\chi^{2}<5$ for a wide range of cutoffs.

There are many possibilities to extend the current study. In particular, the extra parameters provided by the counter terms may be determined in a future work by a fit to properties of some selected finite nuclei. Also, a more conclusive power counting might be drawn after higher-order (e.g., NNLO) contributions are included, which will be addressed in a future work. As an interesting step, it is worth mentioning that the third-order perturbation terms associated to Skyrme forces have been derived recently in Ref. [65].

\section{Acknowledgments}

We thank U. van Kolck for useful discussions and suggestions. This research was supported by the European Union Research and Innovation program Horizon 2020 under grant agreement no. 654002 .

[1] S. Gandolfi, A. Gezerlis, J. Carlson, Ann. Rev. Nucl. Part. Sci. 65, 303 (2015).

[2] B. Friedman and V. R. Pandharipande, Nucl. Phys. A361, 502 (1981).

[3] A. Akmal, V. R. Pandharipande, and D. G. Ravenhall, Phys. Rev. C 58, 1804 (1998).

[4] A. Schwenk and C. J. Pethick, Phys. Rev. Lett. 95, 160401 (2005).

[5] A. Gezerlis and J. Carlson, Phys. Rev. C 77, 032801(R) (2008).

[6] E. Epelbaum, H. Krebs, D. Lee and U. -G. Meissner, Eur. Phys. J. A 40, 199 (2009).

[7] N. Kaiser, Eur. Phys. J. A 48, 148 (2012).

[8] J. Carlson, J. Morales, Jr., V. R. Pandharipande and D. G. Ravenhall, Phys. Rev. C 68, 025802 (2003).

[9] S. Gandolfi, A. Yu. Illarionov, K. E. Schmidt, F. Pederiva and S. Fantoni, Phys. Rev. C 79, 054005 (2009).

[10] A. Gezerlis and J. Carlson, Phys. Rev. C 81, 025803 (2010). 


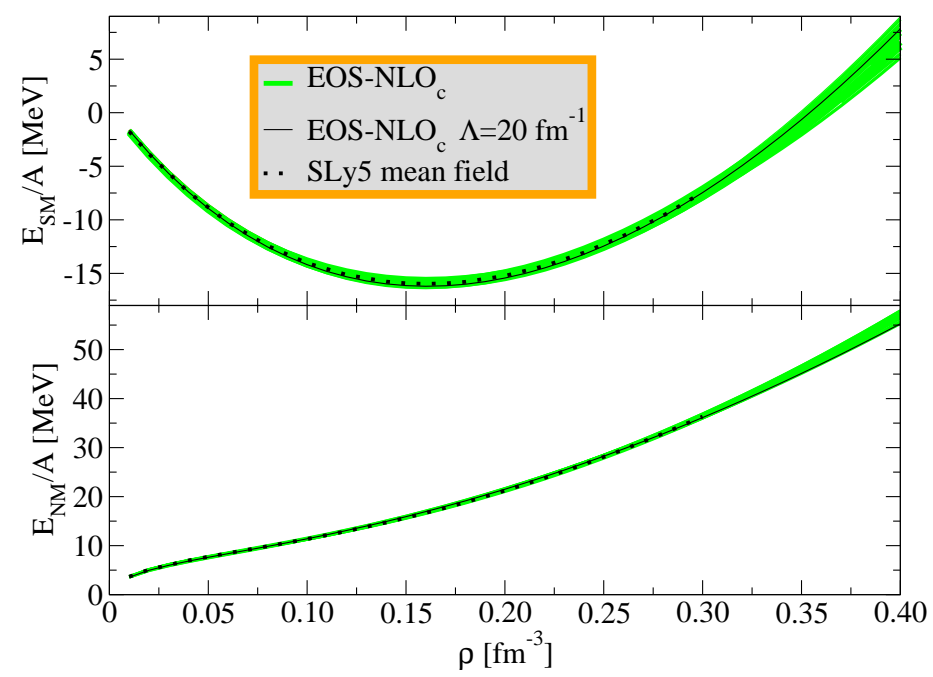

FIG. 8: EOSs as a function of the density $\rho$, where the subscript SN (NM) represents symmetric (neutron) matter. The dotted line (green band) represents the mean-field SLy5 (renormalized NLO) EOSs. The NLO EOSs are obtained by using the scenario (c), that is Eqs. (22) and (23) with the parameters listed in Table VII. Here, the cutoff is taken in the window $\Lambda=1.2-20 \mathrm{fm}^{-1}$ and the errorbars correspond to the cutoff dependence of the fit.

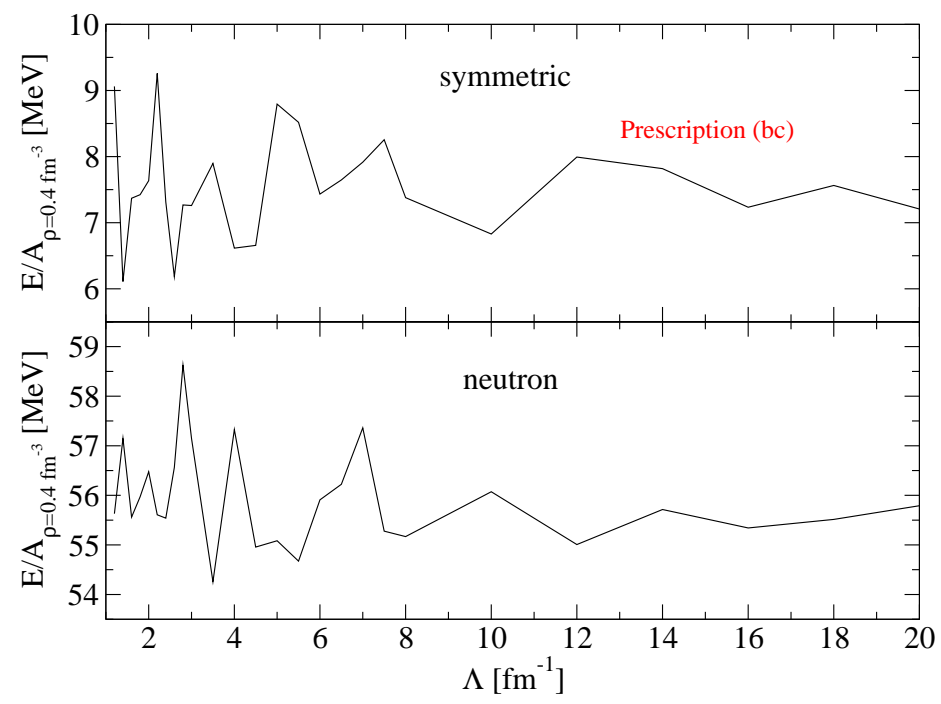

FIG. 9: Second-order EOSs obtained by using Eqs. (18) and (19) [scenario (bc)] at the density value $\rho=0.4$ $\mathrm{fm}^{-3}$, as a function of the cutoff $\Lambda$.

[11] S. Gandolfi, J. Carlson and S. Reddy, Phys. Rev. C 85, 032801 (2012).

[12] M. Baldo, A. Polls, A. Rios, H.-J. Schulze and I. Vidana, Phys. Rev. C 86, 064001 (2012).

[13] K. Hebeler and A. Schwenk, Phys. Rev. C 82, 014314 (2010).

[14] I. Tews, T. Kruger, K. Hebeler and A. Schwenk, Phys. Rev. Lett. 110, 032504 (2013).

[15] A. Gezerlis, I. Tews, E. Epelbaum, S. Gandolfi, K. Hebeler, A. Nogga and A. Schwenk, Phys. Rev. 


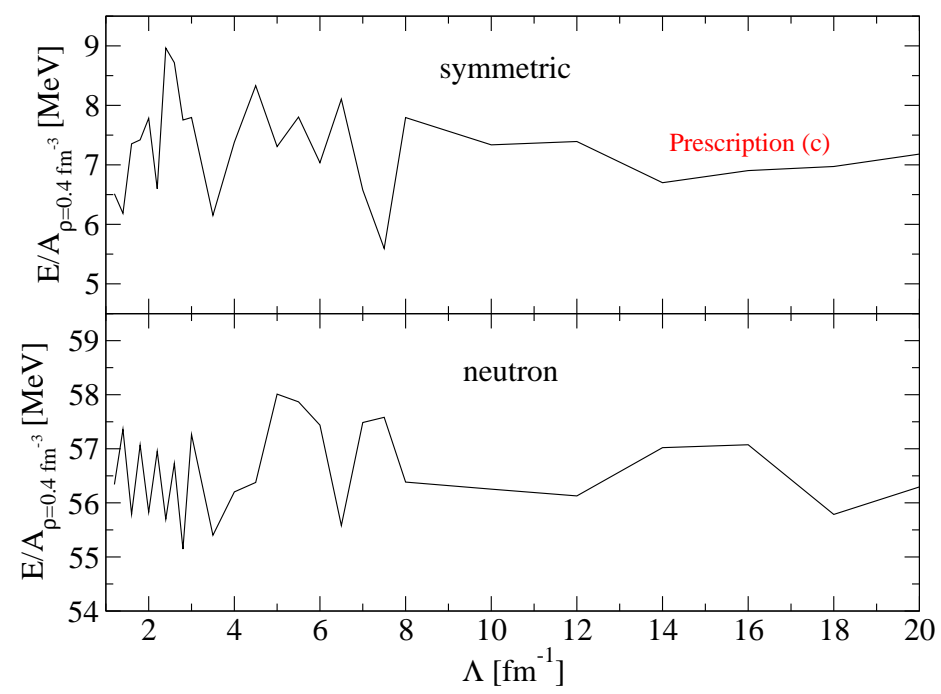

FIG. 10: Second-order EOSs obtained by using Eqs. (22) and (23) [scenario (c)] at the density value $\rho=0.4$ $\mathrm{fm}^{-3}$, as a function of the cutoff $\Lambda$.

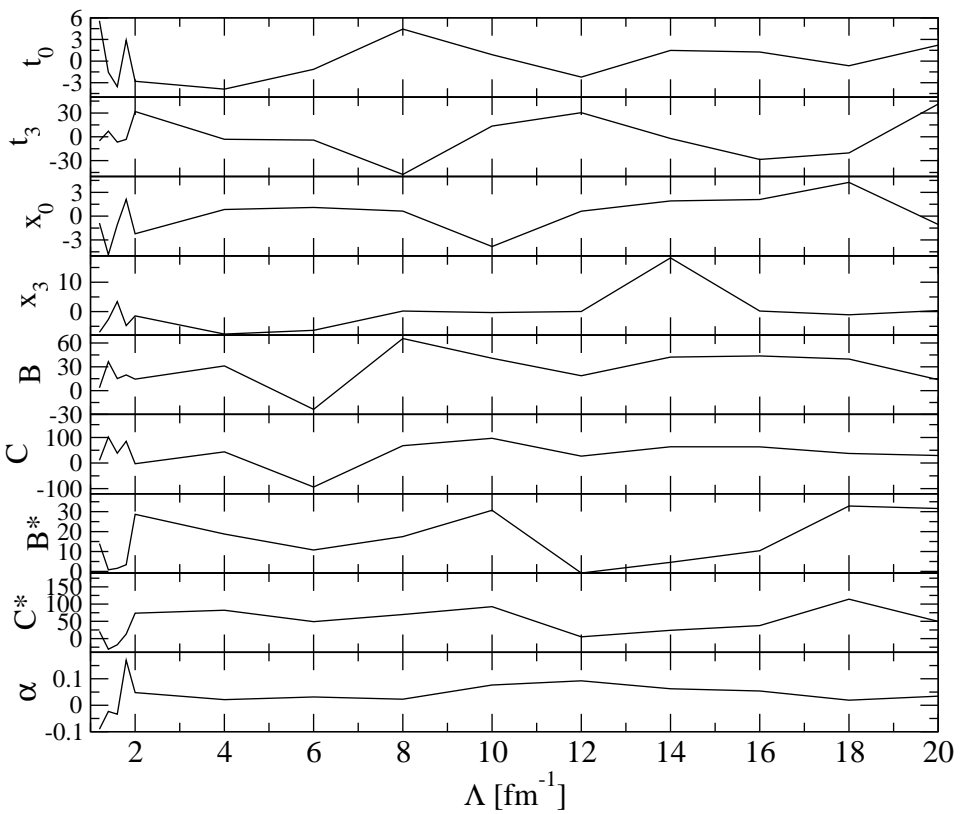

FIG. 11: Parameters in Eqs. (18) and (19) [scenario (bc)] as a function of the cutoff $\Lambda$. Here the units of parameters are those listed in Table VI

Lett. 111, 032501 (2013).

[16] L. Coraggio, J. W. Holt, N. Itaco, R. Machleidt and F. Sammarruca, Phys. Rev. C 87, 014322 (2013).

[17] G. Hagen, T. Papenbrock, A. Ekstrom, K. A. Wendt, G. Baardsen, S. Gandolfi, M. Hjorth-Jensen and C. J. Horowitz, Phys. Rev. C 89, 014319 (2014).

[18] A. Gezerlis, I. Tews, E. Epelbaum, M. Freunek, S. Gandolfi, K. Hebeler, A. Nogga and A. Schwenk, Phys. Rev. C 90, 054323 (2014). 


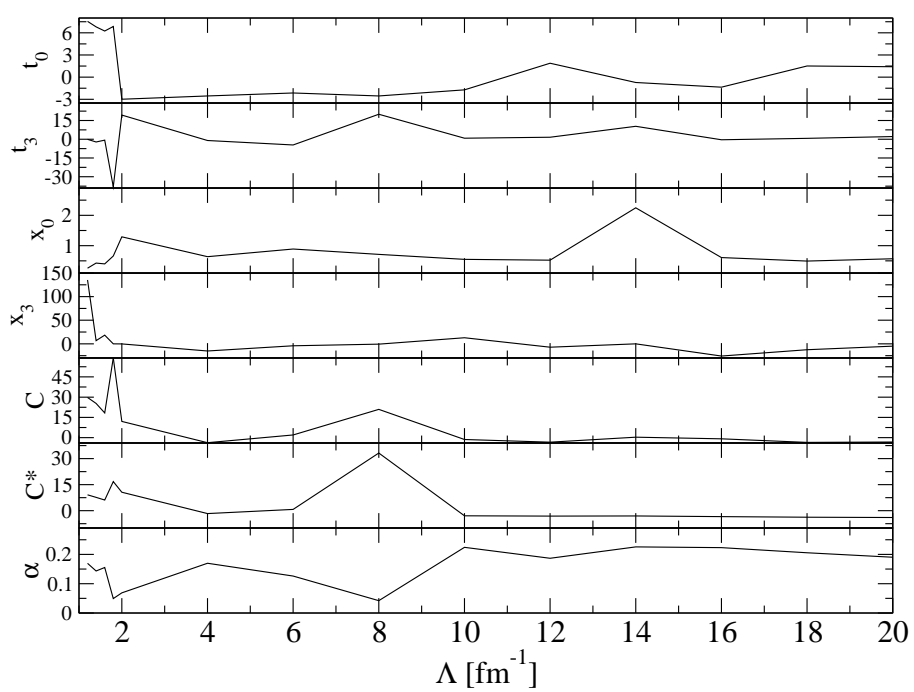

FIG. 12: Parameters in Eqs. (22) and (23) [scenario (c)] as a function of the cutoff $\Lambda$. Here the units of parameters are those listed in Table VII.

[19] A. Carbone, A. Rios and A. Polls, Phys. Rev. C 90, 054322 (2014).

[20] A. Roggero, A. Mukherjee and F. Pederiva, Phys. Rev. Lett. 112, 221103 (2014).

[21] G. Wlazlowski, J. W. Holt, S. Moroz, A. Bulgac and K. J. Roche, Phys. Rev. Lett. 113, 182503 (2014).

[22] J. Lietz, S. Novario, G. R. Jansen, G. Hagen and M. Hjorth-Jensen, Lecture Notes in Physics 936, 293 (2017), "An Advanced Course in Computational Nuclear Physics" Springer International publishing, Editors M. Hjorth-Jensen, M. P. Lombardo, U. van Kolck. arXiv:1611.06765.

[23] S. K. Bogner, H. Hergert, J. D. Holt, A. Schwenk, S. Binder, A. Calci, J. Langhammer, and R. Roth, Phys. Rev. Lett. 113, 142501 (2014).

[24] H. Hergert, S. K. Bogner, T. D. Morris, S. Binder, A. Calci, J. Langhammer, and R. Roth, Phys. Rev. C 90, 041302(R) (2014).

[25] A. Signoracci, T. Duguet, G. Hagen, and G. R. Jansen, Phys. Rev. C 91, 064320 (2015).

[26] E. Gebrerufael, K. Vobig, H. Hergert, and R. Roth, Phys. Rev. Lett. 118, 152503 (2017).

[27] G. R. Jansen, M. D. Schuster, A. Signoracci, G. Hagen, and P. Navrátil, Phys. Rev. C 94, 011301 (2016).

[28] S. R. Stroberg, H. Hergert, J. D. Holt, S. K. Bogner, and A. Schwenk, Phys. Rev. C 93, 051301(R) (2016).

[29] S. R. Stroberg, A. Calci, H. Hergert, J. D. Holt, S. K. Bogner, R. Roth, and A. Schwenk, Phys. Rev. Lett. 118, 032502 (2017).

[30] Alexander Tichai, Eskendr Gebrerufael, Robert Roth, arXiv:1703.05664.

[31] M. Bender, P.H. Heenen and P.G. Reinhard, Rev. Mod. Phys. 75, 121 (2003).

[32] T.H.R. Skyrme, Philos. Mag. 1, 1043 (1956); Nucl. Phys. 9, 615 (1959).

[33] D. Vautherin and D. M. Brink, Phys. Rev. C 5, 626 (1972). 
[34] U. van Kolck, Nucl. Phys. A6 45, 273 (1999); J.-W. Chen, G. Rupak and M. J. Savage, Nucl. Phys. A 653, 386 (1999); J. Gegelia, Phys. Lett. B 429, 227 (1998); M. C. Birse, J. A. McGovern and K. G. Richardson, Phys. Lett. B 464169 (1999); S. R. Beane, P. F. Bedaque, W. C. Haxton, D. R. Phillips and M. J. Savage, Essay for the Festschrift in Honor of Boris Ioffe, in: M. Shifman (Ed.), At the Frontier of Particle Physics, in: Handbook of QCD, Vol. 1, World Scientific, Singapore, 2001, pp. 133-269.

[35] A. Pastore, D. Davesne and J. Navarro, Phys. Rep. 563, 1 (2015).

[36] D. Lacroix, A. Boulet, M. Grasso and C.-J. Yang, Phys. Rev. C 95054306 (2017).

[37] K. Moghrabi, M. Grasso, G. Colo and N.V. Giai, Phys. Rev. Lett. 105, 262501 (2010).

[38] C.-J. Yang, M. Grasso, X. Roca-Maza, and G. Colo and K. Moghrabi, Phys. Rev. C 94, 034311 (2016).

[39] N. Kaiser, J. Phys.G: Nucl. and Part. Phys. 42, 095111 (2015).

[40] K. Moghrabi, arXiv:1607.05829.

[41] C.-J. Yang, M. Grasso, K. Moghrabi and U. van Kolck, Phys. Rev. C 95, 054325 (2017).

[42] T.D. Lee and C.N. Yang, Phys. Rev. 105, 1119 (1957).

[43] H.W. Hammer and R.J. Furnstahl, Nucl. Phys. A 678, 277 (2000).

[44] L. Platter, H.-W. Hammer and Ulf-G. Meissner, Nucl. Phys. A 714, 250-264 (2003).

[45] R.J. Furnstahl, Eft for DFT." Renormalization Group and Effective Field Theory Approaches to Many-Body Systems. Springer Berlin Heidelberg, 2012. 133-191.

[46] S.J. Puglia, A. Bhattacharyya, R.J. Furnstahl, Nucl. Phys. A 723 145-180 (2003).

[47] R.J. Furnstahl, H.-W. Hammer, S.J. Puglia, Annals Phys. 322: 2703-2732 (2007).

[48] R. F. Bishop, Ann. Phys. (NY) 77, 106 (1973).

[49] A.L. Fetter, J.D. Walecka, Quantum Theory of Many-Particle Systems, Dover Publications, Inc., Mineola, New York, 2003.

[50] M. Grasso, D. Lacroix and C.-J. Yang, Phys. Rev. C 95, 054327 (2017).

[51] D. Gogny, Nucl. Phys. A 237, 399 (1975).

[52] J. Decharge and D. Gogny, Phys. Rev. C 21, 1568 (1980).

[53] G.F. Bertsch. 2000. World Scientific. Proceedings of the tenth international conference on recent progress in many-body theories. in Bishop, R., Gernoth, K.A., Walet, N.R., Xian, Y. (eds.) Recent Progress in Many-Body Theories Seattle.

[54] D. Lacroix, Phys. Rev. A 94, 043614 (2016).

[55] P.F. Bedaque, H.-W. Hammer, and U. van Kolck, Nucl. Phys. A 676 (2000) 357; H.-W. Hammer and T. Mehen, Phys. Lett. B 516 (2001) 353; P.F. Bedaque, G. Rupak, H.W. Griehammer, and H.-W. Hammer, Nucl. Phys. A 714 (2003) 589; H.W. Griehammer, Nucl. Phys. A 744 (2004) 192; Nucl. Phys. A 760 (2005) 110; Few-Body Syst. 38 (2006) 67; I.R. Afnan and D.R. Phillips, Phys. Rev. C 69 (2004) 034010; T. Barford and M.C. Birse, J. Phys. A 38 (2005) 697; L. Platter, Phys. Rev. C 74 (2006) 037001.

[56] E. Chabanat, P. Bonche, P. Haensel, and R. Schaeffer, Nucl. Phys. A 627, 710 (1997); ibid. 635, 231 (1998); ibid. 643, 441 (1998). 
[57] B.G. Carlsson, J. Toivanen and U. von Barth, Phys. Rev. C 87, 054303 (2013).

[58] H. Gil, P. Papakonstantinou, C.H. Hyun, T.-S. Park and Y. Oh, Acta Physica Polonica B 48 305, arXiv:1611.04257 [nucl-th].

[59] P. Papakonstantinou, T.-S. Park, Y. Lim, C.H. Hyun, arXiv:1606.04219 [nucl-th].

[60] C. J. Yang, M. Grasso and D. Lacroix, Phys. Rev. C 94, 031301(R) (2016).

[61] T. Schafer, C.-W. Kao and S.R. Cotanch, Nucl. Phys. A 762, 82 (2005).

[62] J. V. Steele, arxiv:nucl-th/0010066v2.

[63] N. Kaiser, Nucl. Phys. A 860, 41 (2011).

[64] H. W. Griesshammer, In 8th International Workshop on Chiral Dynamics (CD) Pisa, Italy, June 29-July 3, CD2015 PoS(CD15)104, arXiv:1511.00490v3 [nucl-th].

[65] N. Kaiser, Eur. Phys. J. A 53, 104 (2017). 